\title{
PROCEEDINGS OF RECENT MEETINGS ON RELATED SUBJECTS
}

1971 Proceedings of the International School of Physics 'Enrico Fermi', course XLVII (ed. by B. K. Sachs), Academic Press, New York

1972 Proceedings of the International School of Physics 'Enrico Fermi' (ed. by B. Bertotti), Academic Press, New York

1972 Les Houches Summer School 'Black Holes; Astres Occlus'

(ed. by Cécile M. DeWitt and B. S. DeWitt),

Gordon and Breach Science Publishers, New York 1973

1972 Texas Symposium on Relativistic Astrophysics

(New York City, December 1972)

Proceedings in press in Proceedings of the New York Academy of Sciences

Colloque International du CNRS no. 220,

'Ondes et radiations gravitationnelles', Paris 1973 